\title{
Targeting PPAR $\gamma$ in the epigenome rescues genetic metabolic defects in mice
}

\author{
Raymond E. Soccio, ${ }^{1,2}$ Zhenghui Li, ${ }^{1,2}$ Eric R. Chen, ${ }^{1,2}$ Yee Hoon Foong, ${ }^{1,2}$ Kiara K. Benson, ${ }^{1,2}$ Joanna R. Dispirito, ${ }^{1,2}$ \\ Shannon E. Mullican, ${ }^{1,2}$ Matthew J. Emmett, ${ }^{1,2}$ Erika R. Briggs, ${ }^{1,2}$ Lindsey C. Peed,, Richard K. Dzeng, ${ }^{1,2}$ Carlos J. Medina, ${ }^{1,2}$ \\ Jennifer F. Jolivert, ${ }^{1,2}$ Megan Kissig,, 2,3 Satyajit R. Rajapurkar,, Manashree Damle,, ${ }^{1,2}$ Hee-Woong Lim, ${ }^{2,4}$ Kyoung-Jae Won, ${ }^{2,4}$ \\ Patrick Seale, 2,3 David J. Steger, ${ }^{1,2}$ and Mitchell A. Lazar ${ }^{1,2}$ \\ 'Department of Medicine, Division of Endocrinology, Diabetes, and Metabolism, ${ }^{2}$ Institute for Diabetes, Obesity, and Metabolism, ${ }^{3}$ Department of Cell and Developmental Biology, \\ and ${ }^{4}$ Department of Genetics, Perelman School of Medicine at the University of Pennsylvania, Philadelphia, Pennsylvania, USA.
}

\begin{abstract}
Obesity causes insulin resistance, and PPAR $\gamma$ ligands such as rosiglitazone are insulin sensitizing, yet the mechanisms remain unclear. In C57BL/6 (B6) mice, obesity induced by a high-fat diet (HFD) has major effects on visceral epididymal adipose tissue (eWAT). Here, we report that HFD-induced obesity in B6 mice also altered the activity of gene regulatory elements and genome-wide occupancy of PPAR $\gamma$. Rosiglitazone treatment restored insulin sensitivity in obese B6 mice, yet, surprisingly, had little effect on gene expression in eWAT. However, in subcutaneous inguinal fat (iWAT), rosiglitazone markedly induced molecular signatures of brown fat, including the key thermogenic gene Ucp1. Obesity-resistant 129S1/SvlmJ mice (129 mice) displayed iWAT browning, even in the absence of rosiglitazone. The 129 Ucp1 locus had increased PPAR $\gamma$ binding and gene expression that were preserved in the iWAT of B6x129 F1-intercrossed mice, with an imbalance favoring the 129-derived alleles, demonstrating a cis-acting genetic difference. Thus, B6 mice have genetically defective Ucp1 expression in iWAT. However, when Ucp1 was activated by rosiglitazone, or by iWAT browning in cold-exposed or young mice, expression of the B6 version of Ucp1 was no longer defective relative to the 129 version, indicating epigenomic rescue. These results provide a framework for understanding how environmental influences like drugs can affect the epigenome and potentially rescue genetically determined disease phenotypes.
\end{abstract}

\section{Introduction}

There is a worldwide epidemic of obesity and diabetes, yet the mechanisms whereby obesity leads to insulin resistance are incompletely understood (1). While diabetes is a multisystem disease also involving skeletal muscle, liver, endocrine pancreas, and other tissues, adipose tissue plays a central and likely initiating role (2). Furthermore, fat distribution matters, as visceral fat correlates with metabolic disease more than does subcutaneous fat (3). A positive energy balance, due to overnutrition and a sedentary lifestyle, results in expansion of adipose tissue mass. While dietary macronutrient content is an area of intense investigation and controversy, high-fat diets (HFDs) have long been implicated in human obesity (4), and HFD feeding of rodents is commonly used to model obesity and its metabolic complications (5).

Inbred mice of different strains have been noted to gain more or less weight on HFD (6-8). C57BL/6 (B6) mice are obesity susceptible, while various 129 strains are resistant, and mouse crosses have identified risk loci but not causative genes or variants (9-11). Even within the most commonly used B6 mice, there are white adipose depot-selective responses to HFD (12). Most studies have focused

Conflict of interest: M.A. Lazar is on scientific advisory boards for Pfizer Inc. and Eli Lilly and Company.

Submitted: October 13, 2016; Accepted: December 30, 2016.

Reference information: J Clin Invest. 2017;127(4):1451-1462.

https://doi.org/10.1172/JCI91211. on visceral fat and noted marked inflammation with macrophage accumulation (13), as well as paradoxically reduced expression of genes involved in lipid storage and metabolism (14-16).

PPAR $\gamma$ is a nuclear receptor transcription factor and a master regulator in adipocytes, and PPAR $\gamma$ agonists, including thiazolodinediones (TZDs) like rosiglitazone, are antidiabetic drugs (17). The exact mechanism for insulin sensitization by rosiglitazone remains elusive, but PPAR $\gamma$ in adipose tissue is the likely target (18), though roles for PPAR $\gamma$ in other tissues like liver, immune cells, and neurons have also been proposed. Furthermore, a recent study even suggests that rosiglitazone reverses HFD-mediated changes in the small intestinal microbiota (19). TZDs have effects on adipocytes, but also increase the number of alternatively activated antiinflammatory macrophages in adipose tissue and reduce markers of inflammation (20).

In white adipose tissue (WAT), there are brown-like adipocytes (sometimes called "beige" or "BRITE") with multiloculated rather than unilocular lipid droplets, abundant mitochondria, and high expression of uncoupling protein 1 (UCP1), which expends energy via uncoupled respiration (21). Importantly, mitochondrial genes in WAT are suppressed by HFD and induced by rosiglitazone $(22,23)$. Furthermore, the weight gain in $\mathrm{B} 6$ mice on HFD is due to decreased energy expenditure, not increased caloric intake, as they consume less of the calorie-dense HFD (14). Therefore, $U c p 1$ expression and browning of white fat may play a role in HFD-induced obesity and insulin resistance. 
A

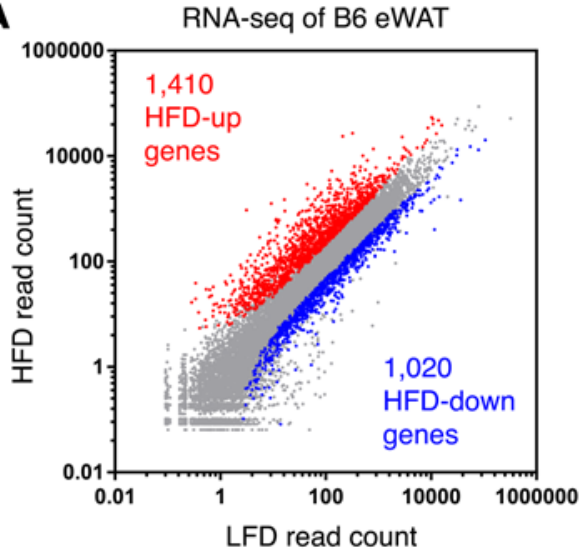

B PANTHER biological process ( $P$ value)

Cytokine- and chemokine-mediated signaling pathway

Cell cycle

Immunity and defense

Cell motility

Mitosis

Cytokine- and chemokine-mediated

immunity

Blood clotting

Signal transduction

Chromosome segregation

Macrophage-mediated immunity

Cell structure and motility

Intracellular signaling cascade

Cell proliferation and differentiation

Amino acid metabolism

Fatty acid metabolism

Lipid, fatty acid and steroid

metabolism

Developmental processes

Synaptic transmission

Carbohydrate metabolism
Figure 1. HFD decreases mRNAs and regulatory elements related to adipocyte metabolism in visceral fat of C57BL/6 mice. (A) Scatterplot of RNA-seq data showing genes regulated by HFD in eWAT, using cutoffs of less than 0.05 for the FDR and a fold-change of greater than 2 in 2 replicate cohorts $(n=4-5$ male B6 mice per diet). (B) Pathway analysis of genes that were HFD-upregulated (HFD-up) (pink) or HFD-downregulated (HFD-down) (blue). (c) ChIP-seq for RNA Pol II in B6 eWAT identified 14,174 sites of intergenic occupancy. Scatterplot shows ChIP-seq for histone acetylation (H3K27ac) at these presumed enhancer sites in eWAT from B6 mice on HFD versus those on LFD. (D and E) HFD-regulated enhancers were analyzed for the occurrence of transcription factor motifs.
C

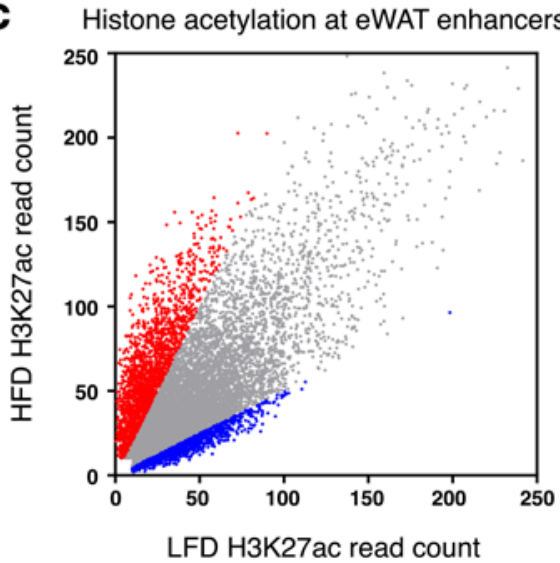

D Macrophage factor motifs

$$
\begin{aligned}
\quad \text { TGAeTCA.- } & \text { AP1 (bZIP, } \left.P=10^{-112}\right) \\
\text { AEAGGAAGTG } & \text { PU.1 (ETS, } \left.P=10^{-107}\right)
\end{aligned}
$$

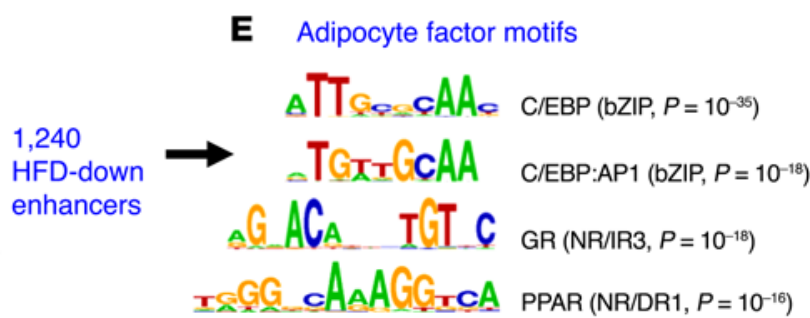

In addition to tissue morphology and gene expression, new methods in the past decade have enabled global profiling of the epigenome through interrogation of chromatin modification and transcription factors affecting DNA function (24). Several epigenomic analyses of obesity and insulin resistance have been undertaken. For instance, changes in hepatic open chromatin mediated by HFD have been profiled (25), and studies of 3T3-L1 adipocytes rendered insulin resistant by glucocorticoids or TNF- $\alpha$ have identified potential roles of nuclear receptors like the glucocorticoid and vitamin D receptors (26). Here, we sought to define epigenomic changes mediated by HFD in adipose tissue by mapping enhancer marks and PPAR $\gamma$ occupancy across the genome. We examined visceral and subcutaneous fat to identify depot-selective effects of HFD in B6 mice, fed the same HFD to 129 mice to find strain differences, and treated mice with rosiglitazone for insulin sensitization. Remarkably, the effects of diet, depot, drug, and strain converged on Ucp1-mediated browning of subcutaneous white fat as a critical metabolic control point. Finally, a genetic defect in Ucp1 gene expression was identified in B6 mice, which could be overcome by treatment with rosiglitazone.

\section{Results}

Diet-induced obesity alters gene-regulatory regions in visceral fat of C57BL/6 mice. Multiple cohorts of male B6 mice were subjected to HFD or a control low-fat diet (LFD) (10\% versus $60 \%$ of calories from fat, respectively) for 12 weeks, resulting in a time-dependent weight gain of approximately $50 \%$, with increased adipose tissue mass and serum leptin levels (Supplemental Figure 1, A-E; supplemental material available online with this article; doi:10.1172/ JCI91211DS1). Since insulin resistance is correlated with visceral adiposity, we first performed RNA-sequencing (RNA-seq) in epididymal white adipose tissue (eWAT), a visceral depot (Figure 1A; RNA-seq details are provided in Supplemental Table 1). As expected, genes indicative of inflammation and macrophages were highly induced by HFD, and expression of adipocyte metabolic genes was decreased by HFD (Figure 1B).

To further probe these changes in gene expression, we took an unbiased approach to identify gene-regulatory regions altered by HFD in B6 eWAT (ChIP-sequencing [ChIP-seq] details are provided in Supplemental Table 2). By using intergenic RNA polymerase II (Pol II) occupancy to identify distal regulatory regions and acetylation at histone $\mathrm{H} 3$ lysine 27 (H3K27ac) to indicate activity, 
A

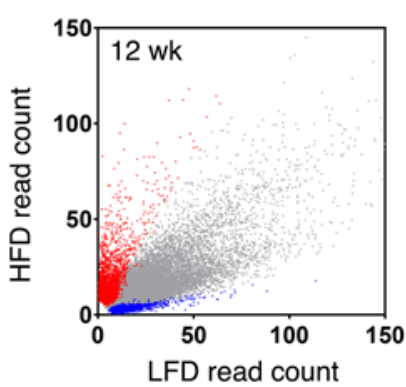

35,878 eWAT

PPAR $y$ sites

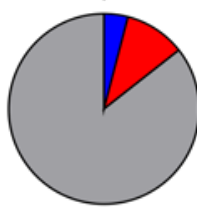

$4 \%$ HFD-down

$10 \%$ HFD-up

$86 \%$ Common
B

26,714 eWAT PPAR $\gamma$ sites in cultured Ads or Macs

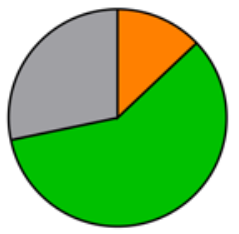

$13 \%$ Macs $59 \%$ Ads $28 \%$ Both

C

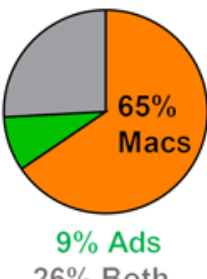

$26 \%$ Both
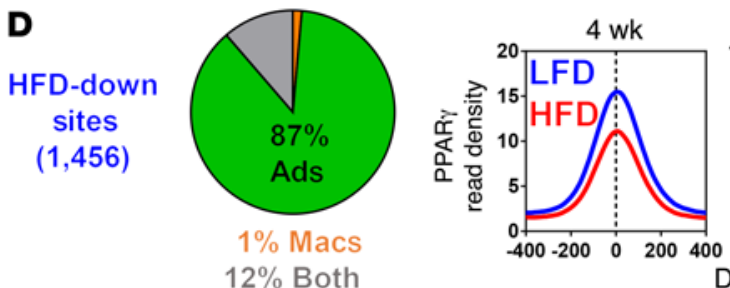

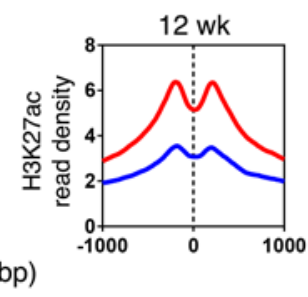

Distance to center (bp)

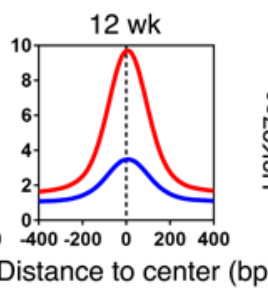

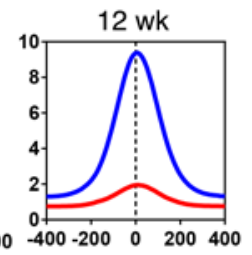

Distance to center (bp)

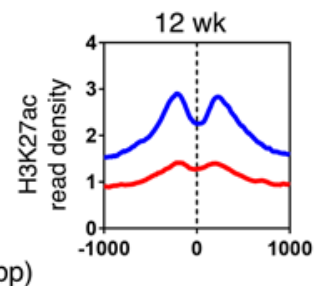

Figure 2. HFD alters PPAR $\gamma$ occupancy and gene regulation in visceral fat of C57BL/6 mice. (A) Scatterplot of approximately 36,000 PPAR $\gamma$ sites identified in 5 ChIP-seq experiments comparing occupancy in eWAT from mice on HFD for 12 weeks versus control mice on LFD. Colored sites indicate a 3 -fold diet-dependent difference in average occupancy, even when corrected for the overall decline on HFD. (B) Of the approximately 36,000 PPAR $\gamma$ sites identified in eWAT, approximately 26,000 were found by ChIP-seq of cultured 3T3-L1 adipocytes (Ads) and/or elicited peritoneal macrophages (Macs), and sites were identified as common versus 4 -fold selective for either cell type. (C) For HFD-upregulated sites from $\mathbf{A}$, there was enrichment of macrophage-selective sites (pie chart). Average profiles show differential PPAR $\gamma$ occupancy at 12 but not 4 weeks of HFD and increased histone acetylation (H3K27ac) at these sites after 12 weeks of HFD. (D) For HFD-downregulated sites from $\mathbf{A}$, there was enrichment of adipocyte-selective sites (pie chart). Average profiles show differential PPAR $\gamma$ occupancy at 12 and 4 weeks of HFD and decreased H3K27ac at these sites on the HFD. (E) Time dependence of H3K27ac changes at HFD-downregulated and HFDupregulated PPAR $\gamma$ sites (error bars indicate the mean and $95 \% \mathrm{CI}$ ). (F) Heatmap sorted by HFD regulation of each PPAR $\gamma$ site, showing whether the nearest gene within $100 \mathrm{~kb}$ was significantly diet regulated.

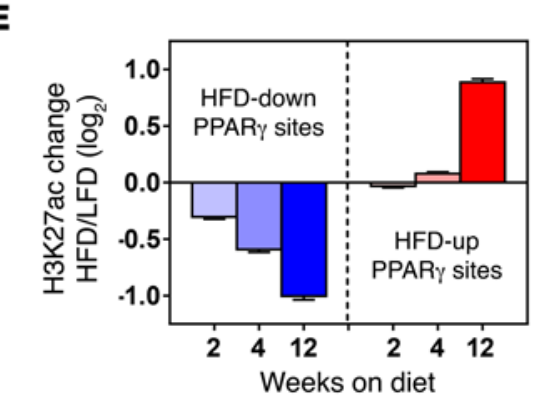

F

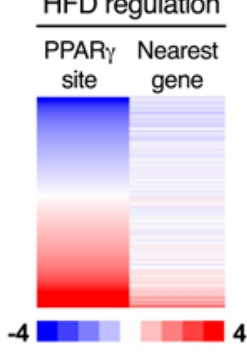

we identified HFD-altered enhancers (Figure 1C). Motif analysis of HFD-upregulated enhancers revealed ETS or bZIP recognition sequences for the top 20-ranked motifs, implicating the macrophage lineage-determining factors PU.1 and AP1 (27), respectively (Figure 1D). The appearance of macrophage enhancers on HFD is consistent with the known increase in macrophages in obese visceral fat as well as the increase in macrophage-related gene expression. Conversely, the top motifs found in HFD-downregulated enhancers implicated the adipocyte lineage-determining factors PPAR $\gamma$ and CCAAT/enhancer-binding protein (C/EBP) and the adipogenic glucocorticoid receptor (GR) (Figure 1E).

$H F D$ remodels PPAR genome-wide binding in visceral fat of $B 6$ mice. Since PPAR $\gamma$ is known as the master regulator of adipocyte biology, we next determined the effects of HFD on the genomic occupancy of PPAR $\gamma$ in visceral fat. Five independent PPAR $\gamma$ ChIPseq experiments using B6 eWAT reproducibly showed a decrease of approximately 2-fold in PPAR $\gamma$ occupancy on HFD (Supplemental Figure 2A). The overall trend for reduced PPAR $\gamma$ occupan- cy may be partially explained by a reduced level of PPAR $\gamma$ mRNA and protein in eWAT of obese mice (Supplemental Figure 2, B and C), which is consistent with earlier reports of reduced Pparg1 and Pparg2 mRNA in eWAT of obese mice (28). Notably, the decline in Pparg mRNA is apparent after only 4 weeks of HFD, before any increase occurs in the expression of macrophage inflammatory markers like EGF-like module-containing mucin-like hormone receptor-like 1 (Emr1) (Supplemental Figure 2B).

Of approximately 36,000 total PPAR $\gamma$ sites, we were able to identify highly diet-selective sites that diverged by at least 3-fold from the overall trend (Figure 2A). We hypothesized that HFD-upregulated sites (opposite of the overall trend) reflect PPAR $\gamma$ binding events in adipose-resident macrophages, which increase in number and PPAR $\gamma$ expression with HFD (29). We previously reported sets of unique PPAR $\gamma$ binding sites in macrophages versus cultured adipocytes (30), and we updated these by performing additional ChIP-seq of thioglycolate-elicited peritoneal macrophages and 3T3-L1 adipocytes, with improved ChIP efficiency 
A
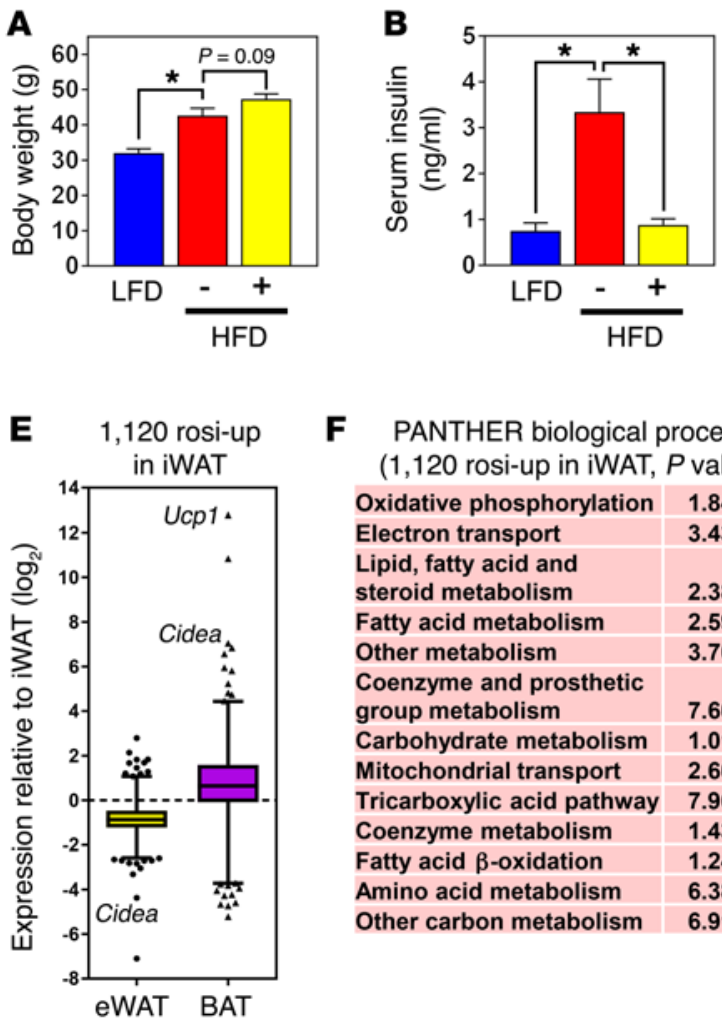

F PANTHER biological process (1,120 rosi-up in iWAT, $P$ value) Oxidative phosphorylation $\quad 1.84 \mathrm{E}-39$ \begin{tabular}{l|l} 
Electron transport & $3.43 \mathrm{E}-39$ \\
\hline
\end{tabular} Lipid, fatty acid and \begin{tabular}{l|l} 
steroid metabolism & $2.38 \mathrm{E}-15$ \\
\hline
\end{tabular} \begin{tabular}{l|l} 
Fatty acid metabolism & $2.59 \mathrm{E}-15$
\end{tabular} \begin{tabular}{l|l}
\hline Other metabolism & $3.76 \mathrm{E}-13$ \\
\hline
\end{tabular} Coenzyme and prosthetic \begin{tabular}{l|l} 
group metabolism & $7.60 \mathrm{E}-13$
\end{tabular} \begin{tabular}{lll} 
Carbohydrate metabolism & $1.01 \mathrm{E}-11$ \\
\hline
\end{tabular} \begin{tabular}{l|l} 
Mitochondrial transport & $2.60 \mathrm{E}-10$
\end{tabular} Tricarboxylic acid pathway $7.96 \mathrm{E}-10$ Coenzyme metabolism $1.43 E-09$ \begin{tabular}{l|l} 
Fatty acid $\beta$-oxidation & $1.24 \mathrm{E}-08$
\end{tabular} \begin{tabular}{lll}
\hline Amino acid metabolism & $6.38 \mathrm{E}-07$ \\
\hline
\end{tabular} Other carbon metabolism $6.91 \mathrm{E}-07$

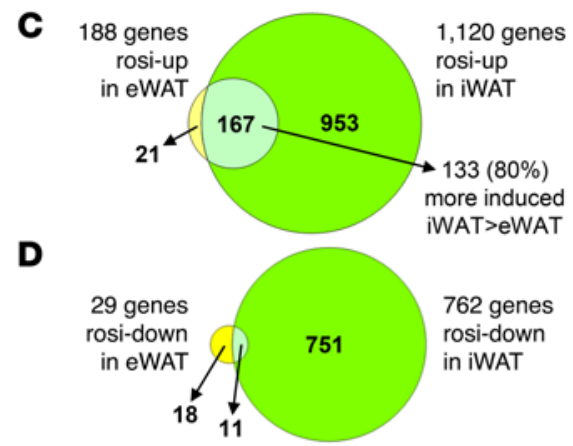

Figure 3. Subcutaneous, but not visceral, fat is markedly responsive to rosiglitazone. B6 mice were subjected to LFD or HFD for 12 weeks or to HFD for 10 weeks, followed by 2 weeks of HFD containing rosiglitazone ( $n=5$ mice per group). (A) Body weights. (B) Random serum insulin levels, with hyperinsulinemia indicating insulin resistance. (C) Venn diagram of RNA-seq results showing the number of genes that were significantly (FDR $<0.05$ ) upregulated by rosiglitazone (rosi-up) in eWAT (yellow) or iWAT (green). (D) Venn diagram showing the number of genes that were downregulated by rosiglitazone (rosi-down) in either or both depots. (E) Box-and-whisker plot of the rosiglitazone-upregulated iWAT genes from $\mathbf{C}$, showing their relative basal expression in eWAT or BAT relative to iWAT. The difference between expression in BAT and eWAT was highly significant $(P<0.0001$, by Wilcoxon matched pairs, signedrank test). (F) Gene ontogeny analysis showing pathways enriched in the rosiglitazone-upregulated gene set. and read depth (Figure 2B). Comparison of these cell data with the eWAT ChIP-seq data revealed that HFD-upregulated PPAR $\gamma$ sites were highly enriched for macrophage-selective binding sites and nearby genes with immune function (Figure $2 \mathrm{C}$ and Supplemental Figure 3A). By contrast, the HFD-downregulated sites were enriched for adipocyte-selective sites and nearby genes involved in lipid metabolism and insulin signaling (Figure 2D and Supplemental Figure 3B).

Interestingly, HFD-induced changes in $\operatorname{PPAR} \gamma$ occupancy occurred with distinct kinetics for up- versus downregulated sites, with HFD-downregulated sites changing earlier (Figure 2, C and D). In agreement with this, the active chromatin mark $\mathrm{H} 3 \mathrm{~K} 27 \mathrm{ac}$, which correlates with PPAR $\gamma$ occupancy (Figure 2, C and D), changed at HFD-downregulated sites earlier than at HFD-upregulated sites (Figure 2E). Moreover, HFD-mediated changes at these sites also correlated with nearest-gene regulation (Figure $2 \mathrm{~F}$ and Supplemental Figure $3 \mathrm{C}$ ), and the degree of regulation correlated with the number of proximal HFD-regulated sites (Supplemental Figure 3D), suggesting that the cistromic changes are functional. In general, HFD-upregulated PPAR $\gamma$ sites were macrophage selective, with HFD-upregulated histone acetylation, and near HFD-upregulated genes, while HFD-downregulated PPAR $\gamma$ sites were adipocyte selective, with HFD-downregulated histone acetylation, and near HFD-downregulated genes (examples are shown in Supplemental Figure 3E). Notably, the adipocyte remodeling indicated by HFD-downregulated $\operatorname{PPAR} \gamma$ sites precedes the appearance of macrophages in great numbers, which is only after 12 or more weeks of $\operatorname{HFD}(31,32)$.

Visceral fat is relatively unresponsive to rosiglitazone. Given the reduced level of PPAR $\gamma$ and its binding sites in visceral WAT on HFD, we were interested in how this depot responds to the antidiabetic drug rosiglitazone, which targets PPAR $\gamma$. As expected, B6 mice on HFD had an increase in body weight of approximately $50 \%$ and marked hyperinsulinemia, indicative of insulin resistance, and after 2 weeks of treatment, rosiglitazone resolved the hyperinsulinemia, despite a trend of increased body weight (Figure 3, A and B). After 2 weeks of rosiglitazone, serum adiponectin levels were also increased, as expected, but the drug had no significant effect on fat pad weights (Supplemental Figure 4, A and B).

Despite causing insulin sensitization, rosiglitazone had little effect on gene expression in eWAT as measured by RNA-seq, with only 188 genes significantly upregulated by the drug (Figure $3 \mathrm{C}$ ), and of these, only 98 increased by more than $50 \%$. Consistent with this finding, PPAR $\gamma$ expression remained reduced in the presence of rosiglitazone (Supplemental Figure 4C), and ChIPseq showed that rosiglitazone had only minimal effects on PPAR $\gamma$ occupancy, slightly reducing HFD-upregulated sites and slightly increasing HFD-downregulated sites (Supplemental Figure 4D), but not bringing them near LFD levels (Supplemental Figure 4E). In addition, while PPAR $\gamma$ agonists have antiinflammatory effects (33), we observed that only 29 genes were significantly reduced by rosiglitazone in eWAT (Figure 3D). Therefore, rosiglitazone had little effect on expression of the HFD-induced macrophage inflammatory genes in eWAT.

Subcutaneous fat is highly responsive to rosiglitazone. Since rosiglitazone had little effect on gene expression in HFD-obese visceral eWAT, we expanded our investigation to study the drug's effects in subcutaneous inguinal WAT (iWAT). Interestingly, the total number of genes regulated by rosiglitazone in iWAT exceeded the total number in eWAT by an order of magnitude (Figure 3, C and D). Even 

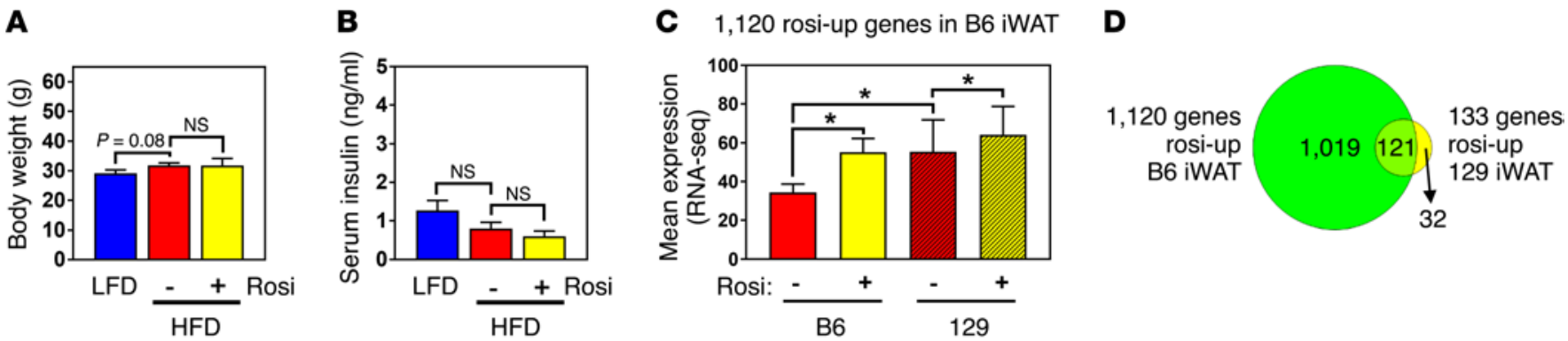

Figure 4. 129 mice show little effect of HFD and have more baseline white fat browning. 129 mice were subjected to LFD or HFD for 12 weeks or to HFD for 10 weeks, followed by 12 weeks of HFD containing rosiglitazone ( $n=5$ mice per group). (A) Body weights. Rosi, rosiglitazone. (B) Random serum insulin levels. (C) RNA-seq expression levels of the rosiglitazone-upregulated genes from B6 iWAT. Mean expression was rosiglitazone-induced in B6 iWAT as expected, but expression was higher in 129 iWAT, even in the absence of rosiglitazone ${ }^{*} P<0.0001$, by Wilcoxon matched pairs, signed-rank test). (D) Venn diagram showing the number of genes that were significantly upregulated by rosiglitazone in 129 iWAT (FDR $<0.05$, yellow) compared with the number of rosiglitazone-upregulated genes in B6 iWAT from Figure 4C (green).

among the 167 genes that were also significantly rosiglitazone activated in eWAT, $80 \%$ showed a greater magnitude of induction in iWAT. Yet, despite these striking effects of rosiglitazone in iWAT, we found that HFD did not alter PPAR $\gamma$-related gene regulation in this depot like it did in eWAT: (a) Pparg expression was not decreased by HFD in iWAT (Supplemental Figure 4C); (b) HFD-downregulated PPAR $\gamma$ sites in eWAT were relatively unchanged by HFD in iWAT (Supplemental Figure 5A); and (c) only less than $10 \%$ of HFD-downregulated adipocyte metabolic genes from eWAT were also HFD downregulated in iWAT (Supplemental Figure 5B). HFD increased macrophage infiltration in iWAT, but at a lower level than in eWAT, as evidenced by increased PPAR $\gamma$ occupancy in iWAT at those HFD-upregulated sites in eWAT (Supplemental Figure 5C), and mildly increased inflammatory gene expression (Supplemental Figure 5D). Furthermore, and similar to eWAT, there was little evidence for antiinflammatory effects of rosiglitazone in iWAT, as the drug did not downregulate the same genes and pathways that were HFD upregulated (Supplemental Figure 5E).

To shed light on the mechanism of rosiglitazone action via subcutaneous fat, we investigated the set of rosiglitazone-induced genes from iWAT. Notably, these genes had lower expression in eWAT, higher expression in interscapular brown adipose tissue (BAT) (Figure 3E), and showed marked enrichment for mitochondrial pathways like oxidative phosphorylation and electron transport (Figure 3F). These properties indicate the appearance of brown-like adipocytes in WAT, which is known to be rosiglitazone induced (34) and to occur preferentially in iWAT (35). Taken as a whole, the data thus far suggest that the insulin-sensitizing effects of TZDs do not occur via eWAT, in which PPAR $\gamma$ levels and gene regulation by rosiglitazone are low, but instead by iWAT, in which there are major changes in gene expression reflecting adipocyte browning.

Subcutaneous fat of rosiglitazone-treated obese $B 6$ mice resembles that of insulin-sensitive 129 mice. Obesity- and diabetes-resistant 129 strain inbred mice are known to have increased browning of WAT compared with B6 mice (36), an effect that may account for some of their resistance to obesity. As expected, 129 mice exposed to the same 12-week HFD regimen that was used in the $\mathrm{B} 6$ experiments displayed only minimal elevations in body weight (Figure 4A and Supplemental Figure 6A), serum leptin (Supplemental Figure 6B), and fat pad weight (Supplemental Figure 6C). Moreover, in con- trast to the striking effects of HFD on B6 eWAT, 129 eWAT showed minimal changes in Pparg expression (Supplemental Figure 6D), PPAR $\gamma$ genome-wide occupancy (Supplemental Figure 6E), and global gene expression (Supplemental Figure 6F). Furthermore, treatment of 129 mice with rosiglitazone elevated serum adiponectin levels (Supplemental Figure 6B) but did not significantly change insulin levels (Figure 4B), similar to the observed effects of rosiglitazone on lean B6 mice. Remarkably, rosiglitazone-induced genes in B6 iWAT had higher basal expression levels in 129 iWAT, such that rosiglitazone administration to obese B6 mice brought the expression of these genes to this higher level (Figure 4C). In fact, far fewer genes were rosiglitazone induced in 129 iWAT (Figure 4D), which may reflect their higher basal expression in 129 iWAT than in B6 iWAT, even in the absence of rosiglitazone.

cis-acting genetic elements drive high basal expression of the Ucp1 gene in 129 mice. The increased brown-like character of 129 iWAT is a genetic difference from the iWAT of B6 mice, and we have shown that rosiglitazone treatment converts B6 iWAT to more closely resemble the basal state of insulin-sensitive 129 mice. The sine qua non of brown fat is $U c p 1$ (37), and we confirmed previous findings that Ucp1 expression levels in iWAT are markedly higher at baseline in 129 mice than in B6 mice (36), with rosiglitazone treatment inducing B6 iWAT Ucp1 expression to reach levels much closer to those detected in 129 iWAT (Figure 5A). Consistent with higher Ucp1 expression, PPAR $\gamma$ binding upstream of the gene was higher in 129 iWAT than in B6 iWAT (Figure 5B and Supplemental Figure 7A). This was true at 5 PPAR $\gamma$ binding sites upstream of Ucp1 and was confirmed by multiple ChIP-seq experiments and ChIP-quantitative PCR (ChIP-qPCR). Remarkably, B6x129 F1 mice showed highly skewed binding of PPAR $\gamma$ to the 129 alleles at all SNPs that differed between the 2 strains (Figure 5C and Supplemental Figure 7B). This was confirmed for several SNPs by ChIP-qPCR, followed by a SNaPshot allele frequency assay (ref. 38 and Figure 5D). Because the 2 alleles co-occupy the same nuclei in F1 iWAT and are therefore equally exposed to trans-acting effects, these data demonstrate that cis effects determine differential binding of PPAR $\gamma$ at the Ucp1 locus.

Previous work identified SNPs at PPAR $\gamma$ binding sites that disrupt transcription factor motifs (39). There are over 400 SNPs that differ between B6 and 129 mice in the approximately 40-kb Ucp1 
A

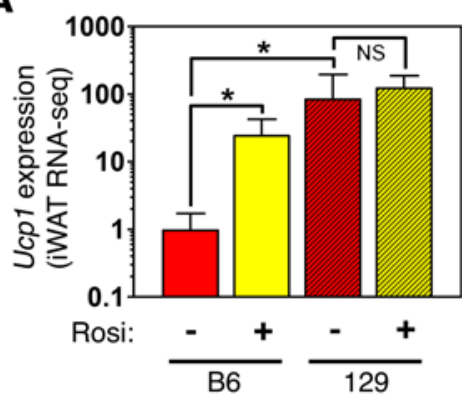

B PPARy ChIP-seq upstream of Ucp1:

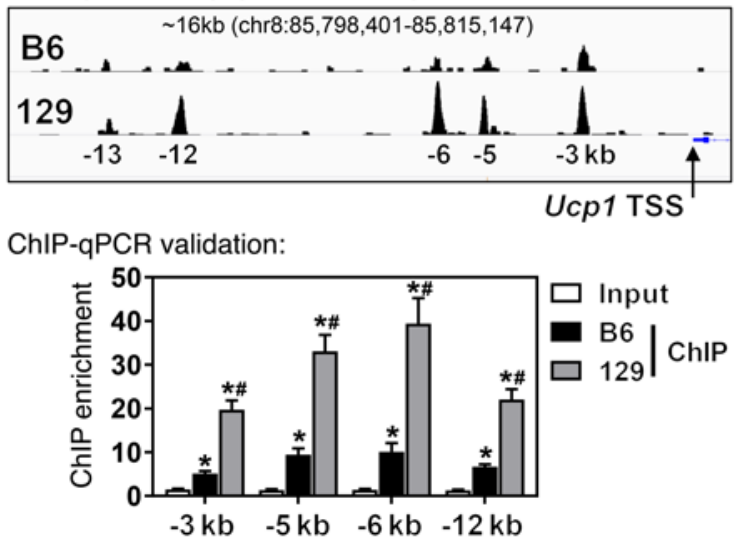

C

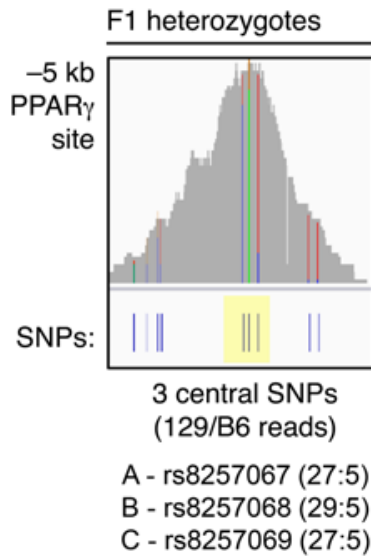

19 SNPs at 5 PPAR $y$ sites

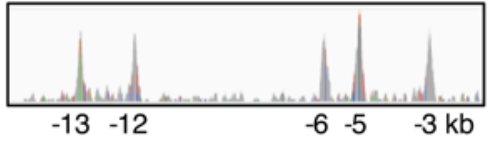

284 total SNP ocurrences in $\mathrm{F} 1 \mathrm{ChIP}$-seq reads:

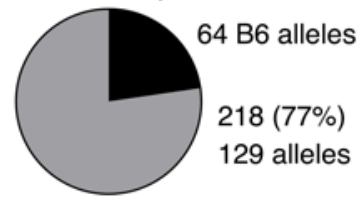

D

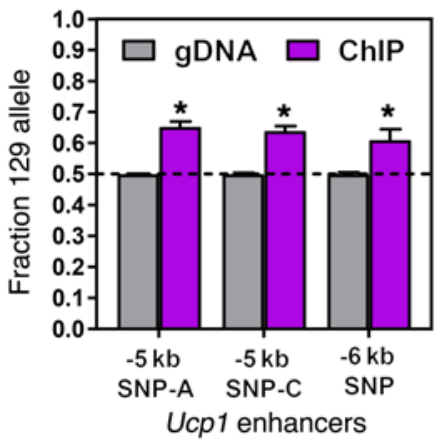

Figure 5. Ucp1 upstream regulatory elements favor PPAR $\gamma$ binding to the 129 locus over the B6 locus. (A) Expression of Ucp1 in B6 and 129 iWAT in the presence and absence of rosiglitazone ( ${ }^{*} P<0.05$, by Mann-Whitney $U$ test). (B) Browser track of PPAR $\gamma$ ChIP-seq at the Ucp 1 locus in B6 versus 129 iWAT, with a graph of ChIP-qPCR confirming stronger binding across multiple sites in 129 iWAT (enrichment vs. negative control site insulin [INS], ${ }^{*} P<0.01$ vs. input, ${ }^{\#} P<0.01$ vs. B6, by $t$ test). chr, chromosome. (C) ChIP-seq in B6x129 F1 mice showing SNPs at PPAR $\gamma$ sites, with an illustration (left) of allelic imbalance at 3 central SNPs at the -5 -kb site. 129 alleles were likewise favored in reads in 19 SNPs at all 5 sites (right). (D) PPAR $\gamma$ ChIP-qPCR products were assayed for imbalance at SNPs using a SNaPshot minisequencing assay, with gDNA showing no imbalance ( $P<0.01$ vs. gDNA, by $t$ test).

locus (Supplemental Figure 7A), and Ucp1 locus regulatory regions have multiple motifs for PPAR $\gamma$ and other collaborative factors (including C/EBP, nuclear factor I [NFI], and early B cell factor [EBF] family members). However, only 2 potential motifs are affected by B6:129 SNPs (Supplemental Figure 7B). Moreover, neither case showed a large allelic effect on consensus motif agreement, and the B6 alleles contained slightly better motifs, even though experimental binding favored 129 alleles (Supplemental Figure 7C).

Imbalanced regulatory elements are predicted to drive imbalanced gene expression, and, indeed, multiple SNPs in the Ucp1 mRNA also showed an allelic imbalance of approximately 4:1 in gene expression favoring 129 alleles in iWAT (Figure 6A). In BAT from the same F1 mice, Ucp1 mRNA expression was not highly imbalanced, thus the $c i s$-acting $U c p 1$ differences appear selective for brown-like adipocytes in iWAT as opposed to classic brown adipocytes in BAT. Importantly, the Ucp1 imbalance was not due to imprinting, as the 129 alleles were overrepresented in F1 iWAT, regardless of the parent of origin, while the maternally imprinted gene Impact showed a 129 or B6 imbalance favoring the paternally derived allele (Figure 6B; note that a similar imbalance was observed in male and female F1 mice). We also measured Ucp1 mRNA imbalance using the gold-standard assay of pyrosequencing. A mixing experiment with different ratios of B6 and 129 genomic DNA (gDNA) demonstrated linearity and precision, and we confirmed a Ucp1 mRNA imbalance in iWAT by this assay (Supplemental Figure 7D). We also examined 16 other genes with expression patterns similar to that of $U c p 1$ (see Supplemental Table 3 , listing polymorphic genes with rosiglitazone upregulation in $\mathrm{B} 6$ iWAT, higher expression in B6 BAT than in B6 iWAT, and higher expression in $129 \mathrm{iWAT}$ than in $\mathrm{B} 6 \mathrm{iWAT}$ ). In contrast to Ucp1, none of these 16 genes had imbalanced mRNA expression in RNA-seq data from F1 iWAT (Supplemental Figure 7E). Therefore, while these other genes are higher in iWAT with browning due to trans effects, $U c p 1$ represents a special case of cis-acting imbalance. Consistent with this finding, we also found strain differential $U c p 1$ expression to be cell autonomous by using cultured primary adipocytes derived from iWAT. We found that 129 cells had 3-fold higher $U c p 1$ expression levels than did $\mathrm{B} 6$ cells, despite similar expression levels of another brown marker, Cidea (Figure 6C), and F1 cells had imbalanced $U c p 1$ expression favoring 129 alleles (Figure 6D). 
A
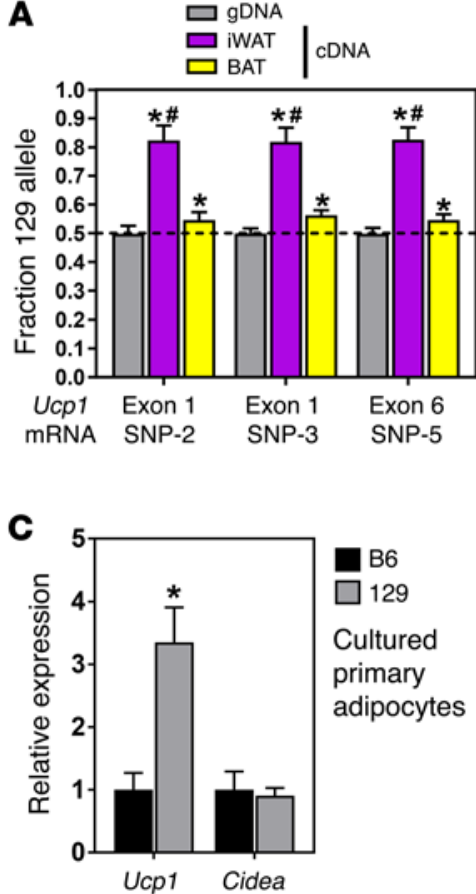

B
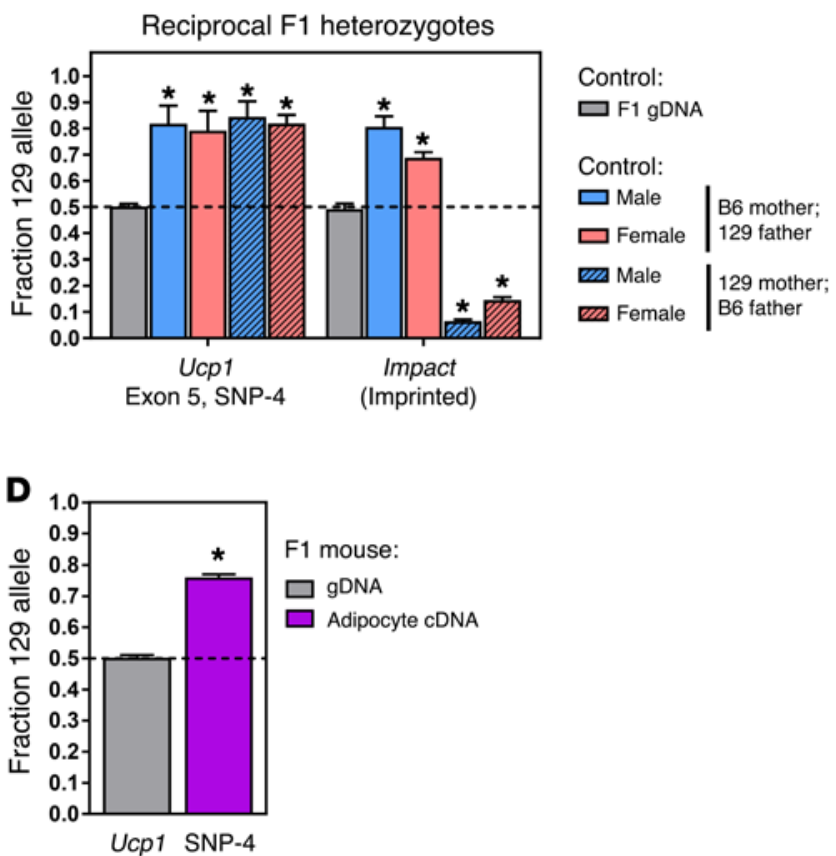

Figure 6. Ucp1 mRNA expression favors 129 over B6 transcripts due to cis-acting effects. (A) To measure allelic imbalance at SNPs in the Ucp1 mRNA, qPCR products were used in SNaPshot assays $\left({ }^{*} P<0.01 \mathrm{vs}\right.$. gDNA, ${ }^{\#} P<0.01 \mathrm{vs}$. BAT, by $t$ test). (B) Male and female F1 mice were generated from reciprocal crosses (B6x129 mice have a B6 mother and 129xB6 mice have a 129 mother), and imbalanced mRNA expression of Ucp1 and the maternally imprinted gene Impact in iWAT were assayed by SNaPshot ( ${ }^{*} P<0.01$ vs. gDNA, by $t$ test). (C) Primary adipocytes were differentiated from stromal vascular fractions of iWAT from B6, 129, and F1 mice. qPCR shows the relative expression of Ucp1 and Cidea normalized to TATA box-binding protein (Tbp) ${ }^{*} P<0.01$ vs. B6, by $t$ test). (D) SNaPshot assay of Ucp1 allelic imbalance in cDNA from F1 primary adipocytes ( $P<0.01$ vs. gDNA, by $t$ test).

Induction of Ucp1 expression rescues the genetic defect in the B6 locus. Having found that cis elements in the 129 Ucp1 gene locus favored expression of that strain's haplotype in the iWAT of 129:B6 F1 mice, we investigated whether the 129 locus was more responsive to rosiglitazone. On the basis of a previous analysis of strain-selective, rosiglitazone-induced genes (39), we anticipated that rosiglitazone might increase the degree of $U c p 1$ imbalance in F1 mice. We found, as expected, that rosiglitazone induced Ucp1 expression in F1 iWAT (Figure 7, A and B), yet evaluation of 6 different SNPs in the Ucp1 mRNA showed that, contrary to expectation, rosiglitazone decreased the degree of imbalance (Figure $7 \mathrm{C}$ ). Also, despite induction of Ucp1 mRNA, the occupancy of PPAR $\gamma$ in B6 iWAT was not altered by rosiglitazone (Supplemental Figure 7A), consistent with previous reports that ligand generally has little or no effect on PPAR $\gamma$ ChIP-seq occupancy at individual sites, but rather alters coregulator recruitment $(40,41)$.

To examine whether other inducers of Ucp1 expression would have the same effect of reducing mRNA imbalance, we turned to the use of cold exposure (Figure 7D). As with the effect of rosiglitazone, cold exposure decreased the degree of allelic imbalance in Ucp1 expression in F1 iWAT, while other cold-induced genes like Dio2 and Ppara showed neither a basal nor a post-induction imbalance (Figure 7E). We also assessed young mice, because normal murine development involves a transient browning of iWAT that peaks at 20 days of age (42), which we confirmed in F1 mice (Figure $7 F)$. Remarkably, imbalanced $U c p 1$ expression in iWAT was reduced in 20-day-old mice relative to imbalance in adult mice (Figure 7G).
Taken together, the data show that induced Ucp1 expression in iWAT of F1 mice, whether by rosiglitazone, cold exposure, or development, normalizes transcript levels between B6 and 129 alleles, thus overcoming the genetic defect in $\mathrm{B} 6$ mice.

\section{Discussion}

We found that HFD-induced obesity and insulin resistance in B6 mice were associated with major derangements in visceral fat including changes in PPAR $\gamma$ and its gene regulation. Treatment with the PPAR $\gamma$ activator rosiglitazone was insulin sensitizing, yet had little effect on visceral fat and instead had major effects on subcutaneous fat, indicative of adipocyte browning. The 129 mice were resistant to HFD-induced obesity, and their subcutaneous fat already had high levels of browning, even without drug treatment. The Ucp 1 gene mediates energy dissipation by brown fat, and its differential expression in 129 mice compared with B6 mice was remarkably preserved as an imbalance between the B6 and 129 loci in F1 intercrossed mice, indicating cis-acting effects. However, upon stimulation of browning with rosiglitazone, the B6 Ucp1 locus had activity similar to that of the 129 Ucp1 locus, showing that the genetic defect in Ucp1 expression can be rescued by drug treatment.

Our finding of HFD-induced inflammatory gene expression in eWAT is consistent with previous reports of macrophage expansion in obesity $(13,29)$. Here, we show for the first time to our knowledge that the cistromic signature of resident macrophages is detectable by ChIPseq in whole adipose tissue. Notably, however, adipocyte PPAR $\gamma$-related changes in the epigenome preceded macrophage effects, and rosigl- 
A

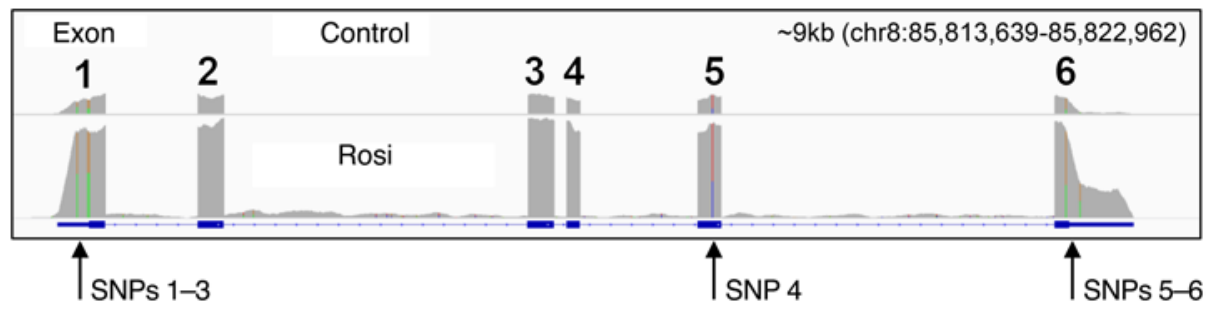

B

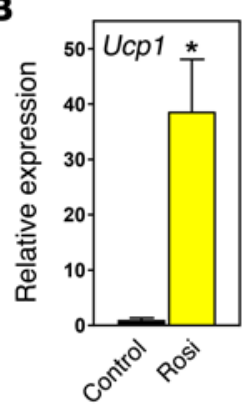

D
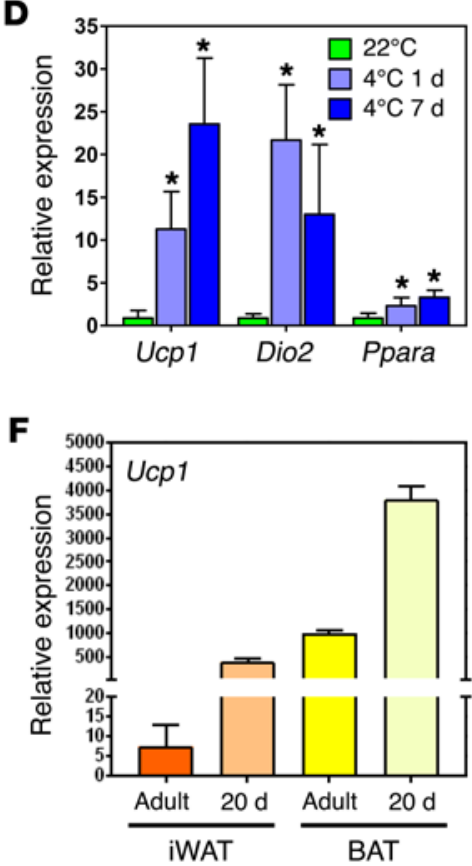

C

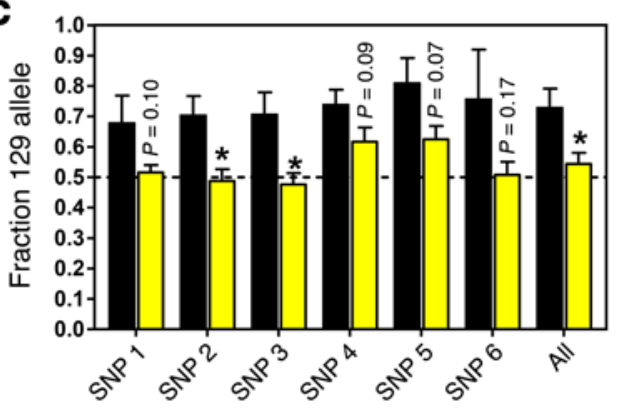

$\mathbf{E}$

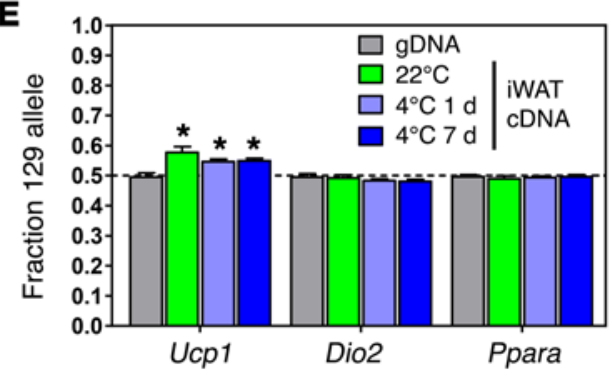

G

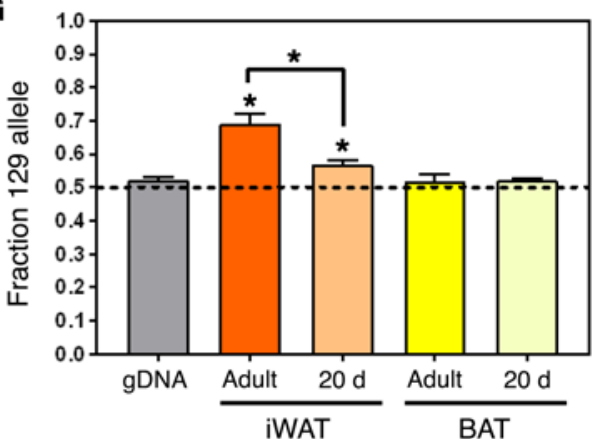

Figure 7. Rosiglitazone-, cold-, and development-induced browning rescue the $B 6$ genetic predisposition to low Ucp1 expression. (A) Browser track of the Ucp1 gene, with F1 iWAT RNA-seq reads in 6 exons and 6 B6:129 SNPs in the mRNA. (B) qPCR confirming that Ucp1 expression is increased by rosiglitazone in this F1 iWAT ( $n=5$ each).

(C) Allelic imbalance in RNA-seq reads at these 6 SNPs in control or rosiglitazone-treated mice ( $n=5$ each). An imbalance was present in control mice but reduced by rosiglitazone treatment. (D) F1 mice were housed at $22^{\circ} \mathrm{C}$ for 1 week, then exposed to cold at $4^{\circ} \mathrm{C}$ for 1 day or 1 week. qPCR shows induced gene expression. (E) A SNaPshot allelic imbalance assay was performed on SNPs in these qRT-PCR products. (F) IWAT and BAT from young 20-day-old mice was compared with iWAT and BAT from 12-week-old adults for Ucp1 expression by qRT-PCR. (G) Imbalanced Ucp1 expression in these cDNAs was assayed by pyrosequencing. ${ }^{*} P<0.05$ versus control or gDNA, by $t$ test. itazone treatment was insulin sensitizing, despite failing to reduce this inflammatory macrophage signature. There is a rich literature on the antiinflammatory effects of TZDs like rosiglitazone (33), yet in other studies that typically involved longer TZD treatment of obese mice, the decline in inflammatory macrophage markers was often small (less than 2-fold) relative to a large induction of such genes in obesity $(31,43-45)$. Indeed, a recent study blocked HFD-induced adipose tissue macrophage recruitment, yet HFD still caused the same degree of obesity and insulin resistance (46). Likewise, although PPAR $\gamma$ is a key regulator of macrophage phenotype (47), mice with transplanted macrophages lacking PPAR $\gamma$ showed the same insulin resistance on HFD and the same TZD-induced insulin sensitization (48). Overall, the role of inflammation as a cause as opposed to a consequence of insulin resistance remains uncertain (49).
It seems paradoxical that obesity reduces visceral fat expression of PPAR $\gamma$ and its target genes involved in lipid storage, but this has been a consistent finding across studies (14-16). The pronounced HFD-induced changes in PPAR $\gamma$ occupancy and gene expression in eWAT of B6 mice are probably the effects of obesity itself on this depot, rather than of the HFD per se, as the effects were less marked in the iWAT of B6 mice and virtually absent in the eWAT or iWAT of 129 mice that remained lean on the same HFD. Therefore, the changes in B6 eWAT are not due simply to a component of the HFD but rather to the depot- and strain-specific effects of this diet. It remains unclear whether this is a signature of adipocyte dysfunction, or instead an adaptive response to overnutrition. Studies of cultured mouse 3T3L1 adipocytes have shown that Pparg expression is repressed by 
inflammatory cytokines like TNF- $\alpha$ (50) or by ATF3 (51), a factor we showed to be markedly HFD upregulated in eWAT, and these could explain the downregulation of Pparg in visceral fat on HFD. We suggest that decreased PPAR $\gamma$ levels in visceral fat on HFD may be responsible for the reduced adipocyte-specific gene expression and may also explain why TZDs have much more pronounced effects on subcutaneous fat than on visceral fat in mice (52). In support of this hypothesis, human cultured primary adipocytes derived from subcutaneous, but not visceral, WAT responded robustly to rosiglitazone (53).

The browning of white fat by rosiglitazone, cold exposure, and other stimuli involves cell differentiation, with neural and even immunological mechanisms triggering transcription factors important for brown-like adipocytes such as PRDM16, FOXC2, and EBF2 (54). It has long been noted that mouse strains differ in their degree of browning in white fat depots. Early studies showed higher Ucp1 expression in A/J mice than in B6 mice (55), and further studies have extended this to show that 129 strains have likewise higher expression levels of Ucp1 than do B6 strains $(36,56)$. Importantly, the genetic difference in $U c p 1$ expression is selective for white but not brown fat (57), leading to the idea that "fundamentally different mechanisms for regulating Ucp1 expression must exist for the brown adipocytes in the traditional white fat vs. brown fat depots" (58). Indeed, brown-like adipocytes have distinct developmental origins from those of classic brown adipocytes (59). Mouse genetic studies have attempted to dissect the loci that determine WAT Ucp1 expression (60), and though signal was found on chromosome 8 near the Ucp1 gene, indicating potential cis effects, the focus has been on trans-acting loci on other chromosomes. Studies of cultured adipocytes from B6 and 129 mice have also suggested trans-acting factors that regulate the development of brown-like adipocytes (61). Thus, there is a consensus that trans effects generally drive brown-like adipocyte differentiation and thus expression of $U c p 1$ and other genes.

By studying allelic imbalances at SNPs in F1 heterozygous mice, we show for the first time to our knowledge that the Ucp1 locus has a cis-acting imbalance favoring 129 SNP alleles over B6 alleles, and this was apparent in both PPAR $\gamma$ binding at enhancer SNPs and in mRNA expression at exonic SNPs. We validated these surprising findings in multiple mouse cohorts, using several independent methods to assay imbalance at multiple SNPs, with results consistently favoring 129 alleles over B6 alleles. While trans effects could account for some of the difference in browning between 129 and B6 mice, a cis effect is the only explanation for imbalanced Ucp1 expression in F1 iWAT, showing genetically defective mRNA expression from the B6 locus. While differences among strains in PPAR $\gamma$ binding are often related to SNPs that alter transcription factor motifs (39), these were not apparent in Ucp1 PPAR $\gamma$ binding sites. Furthermore, rather than just one site, the whole cluster of regulatory regions showed strain-selective PPAR $\gamma$ binding. Perhaps one or more genetic variants are causal and drive differences across many kilobases, and further mechanistic dissection will test this hypothesis. Importantly, imbalanced $U c p 1$ expression was selective for white subcutaneous fat, not interscapular brown fat, in agreement with the idea that fundamentally different mechanisms control Ucp1 expression in white fat compared with expression in brown fat. Most remarkably, inducers of Ucp1 expression like rosiglitazone or cold exposure normalized the imbalance between the B6 and 129 alleles in iWAT of F1 mice. This involved an epigenomic change, as it did not affect the B6 DNA sequence, but instead increased transcriptional activity (without even affecting PPAR $\gamma$ occupancy) at the previously defective Ucp1 locus.

Given that genetic and pharmacological manipulation of Ucp1 generally supports a beneficial role in metabolic disease (37), variable Ucp1 expression may contribute to genetic differences in obesity and diabetes. Mouse genetic studies have identified obesity risk loci by crossing the same B6 and 129 mice used here, and the Obq31 obesity risk peak on chromosome 8 overlies the Ucp1 gene, leading the authors to identify $U c p 1$ as the best obesity candidate among 35 genes in the region (10). In humans, a common polymorphism upstream of UCP1 has been identified and correlated with mRNA abundance (62). This is analogous to what we report in inbred mice, and in both species it remains to be determined which variants are causal as opposed to linked. Notably, multiple small, targeted human genetic studies have associated UCP1 upstream polymorphisms with obesity (63-66), energy expenditure (67), age-dependent BAT activity on PET scans (68), and even longevity (69). However, these positive studies must be interpreted with caution, as meta-analyses failed to show associations with BMI (70), and UCP1 has not emerged as an obesity locus in GWAS (71). Nonetheless, both mouse and human genetics may implicate UCP1 in metabolic disease, and here we demonstrate that genetically determined low Ucp1 expression levels can be overcome by the environmental effects of drug or cold exposure. This is relevant not only to UCP1 and metabolic diseases, but for any complex genetic disease, since most risk alleles are thought to function as regulatory variants affecting gene expression (72). It is therefore a hopeful message that genes are not always destiny and that environment can rescue genetic defects in gene expression by affecting the epigenome.

\section{Methods}

Animals. WT inbred C57Bl/6J and 129S1/SvImJ mice and F1 intercrossed progeny (B6129SF1/J) were purchased from The Jackson Laboratory. Mice were housed, 5 per cage, in a temperature-controlled, specific pathogen-free facility with a 12-hour light/12-hour dark cyle and ad libitum access to water and food. Mice were bred and weaned on standard rodent chow, and starting at 6 weeks of age, mice were fed a purified-ingredient OpenSource diet: HFD (60:20:20 kcal percentage of fat/carbohydrate/protein) or a matched LFD (10:70:20 kcal percentage of fat/carbohydrate/protein) (Research Diets D12492 [HFD], D12450B [LDF]). After 2, 4, or 12 weeks on the diet, mice were euthanized between 4:00 and 5:00 pm by $\mathrm{CO}_{2}$ asphyxiation, followed by cervical dislocation. The epididymal and inguinal white fat pads and interscapular brown fat pads were dissected and snap-frozen in liquid $\mathrm{N}_{2}$. For drug treatment, rosiglitazone (Cayman Chemicals) was incorporated into the diets by Research Diets at $36 \mathrm{mg} / \mathrm{kg}$ of diet, such that a 30 -gm mouse eating $3 \mathrm{gm}$ of diet per day received a rosiglitazone dose of $3.6 \mathrm{mg} / \mathrm{kg} / \mathrm{d}$. B6 or 129 mice were fed HFD for 10 weeks and the rosiglitazone-containing HFD or the control HFD for the final 2 weeks. F1 mice received 2 weeks of the control LFD or the rosiglitazone-containing LFD. 
ChIP-seq. ChIP-seq of adipose tissue was performed as previously described (39). The antibodies used were against PPAR $\gamma$ (Santa Cruz Biotechnology Inc.; catalog sc-7196; $10 \mu$ g per immunoprecipitation); H3K27ac (Abcam; catalog ab4729; $5 \mu \mathrm{g}$ ); and RNA Pol II (Santa Cruz Biotechnology Inc.; catalog sc-899; $5 \mu \mathrm{g}$ ). Reads were aligned to the $\mathrm{mm} 9$ reference genome using Bowtie (73). Peak identification and quantification were performed using the HOMER (Hypergeometric Optimization of Motif EnRichment) software suite (27), and heatmaps were generated using Java Treeview (74). H3K27ac and RNA Pol II ChIP-seqs were performed on B6 cohort 4 (see Supplemental Figures 1 and 6 for mouse cohorts) to analyze the effects of a 12-week HFD, and H3K27ac ChIP-seq on cohort 1 for the effects of HFD at 2 and 4 weeks. To identify enhancers, peaks of Pol II occupancy were identified in intergenic regions on the basis of HOMER annotation, and $\mathrm{H} 3 \mathrm{~K} 27 \mathrm{ac}$ reads were counted in 1,000 bp around each peak center for the HFD and LFD. Diet-selective enhancers were defined by a 2-fold difference in $\mathrm{H} 3 \mathrm{~K} 27 \mathrm{ac}$ reads between diets, and enriched motifs were identified using HOMER. PPAR $\gamma$ ChIP-seq of B6 eWAT comparing 12 weeks of HFD with LFD was performed 5 times (experiment nos. 1 and 2 on B6 cohort 4; no. 3 on cohort 3; and nos. 4 and 5 on cohort 5). The universe of 35,878 binding sites was generated by merging peak calls and requiring a minimum height of 1 read per million (rpm) on at least 1 diet in 3 or more of the 5 experiments. To identify diet-selective peaks, PPAR $\gamma$ tag counting was performed within the central $100 \mathrm{bp}$ of each peak, and the HFD/LFD $\log _{2}$ ratio was calculated. Each experiment had an overall skew toward higher PPAR $\gamma$ binding on LFD (Supplemental Figure 2A), and this skew was defined by the mean of the mid-50\% distribution of the HFD/LFD ratios. The HFD/LFD ratio in each experiment was corrected for this skew, and diet-selective peaks were defined by having a mean corrected ratio of a greater than 3-fold diet difference across all 5 experiments. Genomic Regions Enrichment of Annotations Tool (GREAT) was used to associate biological pathways with binding sites (75). To compare the effects of a 4-week HFD on PPAR $\gamma$ occupancy in B6 eWAT, ChIP-seq was performed on cohort 2. To assay the effects in the B6 iWAT depot at 12 weeks, mice from cohort 3 were used. Cohort 5 included rosiglitazone-treated mice in order to determine the effects of rosiglitazone on PPAR $\gamma$ occupancy. For PPAR $\gamma$ ChIP-seq of eWAT from 129 mice on HFD for 12 weeks, mice from 129 cohort 1 were used. Motif identification in Ucp1 regulatory regions was performed using HOMER or MEME Suite MAST software (76), scanning for motifs in both B6 and 129 sequences.

RNA-seq. Libraries were prepared and sequenced as previously described (39). To initially determine the effects of HFD versus LFD on eWAT (Figure 1A and Supplementary Figure 6F), RNA-seq was performed on eWAT from B6 cohort 4 and 129 cohort 1 . To determine the differential effects on various fat depots of LFD, HFD, and HFD with rosiglitazone (all other figures), RNA-seq was performed on eWAT, iWAT, and/or BAT from B6 cohort 5 and 129 cohort 2. All RNA-seq had 4-5 mice per group. Raw reads were aligned to the $\mathrm{mm} 9$ reference genome using Tophat version 2.1.0 using the parameters recommended by the original authors (77). Gene level quantification was performed by HTSeq (78) using RefSeq genes as a reference and default parameters. Differential expression analysis was done using DESeq2 according to the original authors' instructions (79). To select genes regulated by HFD, we required an FDR of less than 0.05 and a fold-change of more than 2 in both cohorts. To select rosiglitazone-regulated genes, we required an FDR of less than 0.05. For regulated gene lists, Gene Ontology Biological Processes (GOBP) enrichment was performed using Database for Annotation, Visualization, and Integrated Discovery (DAVID) software with a whole-genome background (80). RNA-seq gene expression was also integrated with PPAR $\gamma$ binding sites by ChIP-seq, with each site assigned to the nearest gene's transcription start site (TSS) within $100 \mathrm{~kb}$, or, for each gene, the nearby diet-selective sites were tallied within $100 \mathrm{~kb}$ of the TSS in either direction.

Allelic imbalance assays. SNPs differing between B6 and 129 mice were derived from published genome sequencing (81). SNaPshot (Applied Biosystems) mini-sequencing assays were performed as previously described (39) to measure SNP allele frequency (38). Pyrosequencing allele quantification primers were designed used PyroMark Assay Design software (QIAGEN), with 2 PCR primers (1 biotinylated for capture of single-stranded product) and 1 pyrosequencing primer. PCR was performed using the PyroMark PCR kit and pyrosequencing with PyroMark Gold reagents on a PyroMark Q96 MD instrument (all from QIAGEN) according to the manufacturer's instructions. To assay allelic imbalance in ChIP-seq or RNA-seq reads, BAM files were generated using SAM tools (82) lacking any duplicate reads, and alleles were tallied at SNP positions using the Integrated Genomics Viewer (Broad Institute) (83).

Western blot analysis. B6 eWAT samples from cohort 3 were used. Protein extract $(23 \mu \mathrm{g})$ in RIPA buffer was loaded into each lane of a $10 \%$ polyacrylamide gel (Mini-Protean TGX; Bio-Rad), transferred overnight onto PVDF (Immunobilon-P; EMD Millipore), and blocked for 1 hour in TBST with 5\% milk and 0.05\% Tween-20. For anti-PPAR $\gamma$ Western blotting, the primary PPAR $\gamma$ antibody (1:1,000; Santa Cruz Biotechnology Inc.; catalog sc-7196) was incubated overnight at $4^{\circ} \mathrm{C}$, and the secondary anti-rabbit antibody for 1 hour (1:2,000; ECL Rabbit IgG; GE Amersham). For the anti-Ran loading control, primary anti-Ran antibody (1:2,500; BD Biosciences; cata$\log 610340)$ was incubated for 1 hour at room temperature and the secondary anti-mouse antibody for 1 hour (1:5,000; GE Amersham; ECL Mouse IgG). After the primary antibody incubation, the gels were washed 3 times in TBST with $0.1 \%$ Tween-20 and washed again 3 times in TBST after the secondary antibody incubation. Signal was developed using Western Lightning Plus ECL Reagent (PerkinElmer) and visualized on a Gel Doc (Bio-Rad).

Other methods. Serum was prepared from mice in B6 cohort 5 and 129 cohort 2, and well-validated ELISA assays were used to measure levels of insulin (ALPCO), leptin, and adiponectin (Linco). Mouse 3T3L1 adipocytes and elicited peritoneal macrophages from $\mathrm{B} 6$ mice were cultured as previously described (30). Primary mouse adipocytes were differentiated from the stromal vascular fraction of inguinal fat pads as previously described (84). qPCR was performed using the Applied Biosystems 7500 Fast Real-Time PCR System and Power SYBR Green PCR Master Mix (also from Applied Biosystems). All primer sequences are listed in Supplemental Table 4.

Data deposition. All next-generation sequencing data (ChIP-seq and RNA-seq) were deposited in the NCBI's Gene Expression Omnibus (GEO) database (GEO GSE91067).

Statistics. GraphPad Prism, version 7.01 (GraphPad Software) was used for graphing and statistical tests. Error bars represent the SEM, and statistical significance was determined by 2-tailed, type $2 t$ test, with a $P$ value of less than 0.05 considered significant, unless otherwise stated in the figure legends. 
Study approval. All mouse care and use procedures were approved by the IACUC of the University of Pennsylvania.

\section{Author contributions}

RES and MAL conceived all studies, with help from DJS and SEM. Most of the experiments were designed by RES and performed by ERC, YHF, and KKB. Additional experiments were conducted by JRD (macrophage and 3T3-L1 ChIP-seq); DJS (H3K27ac and Pol2 ChIP-seq); MJE (cold exposure); MK and PS (primary adipocyte culture); CJM and JFJ (some Ucp1 studies); and ERB, LCP, and RKD (animal husbandry and other assays). Computational analyses of RNA-seq were done by ZL and ChIP-seq by RES, with help from SRR, MD, HWL, and KJW. The manuscript was drafted by RES and MAL and revised and approved by all authors.

\section{Acknowledgments}

We thank the Functional Genomics Core and Radioimmunoassay Biomarkers Core of the Penn Diabetes Research Center (P30DK19525), and the laboratory of Marisa Bartolomei (University of Pennsylvania) for use of a pyrosequencer. This work was supported by the JPB Foundation (to MAL) as well as by NIH grants (R01-
DK49780, to MAL; K08-DK094968, to RES; R01-DK098542, to DJS; R01-DK10300802, to PS; and R01-DK106027, to KJW).

Address correspondence to: Raymond Soccio or Mitchell Lazar, 3400 Civic Center Boulevard, Smilow Center for Translational Research 12th floor, Philadelphia, Pennsulvania 19104, USA. Phone: 215.573.4576; E-mail: soccio@mail.med.upenn.edu (R. Soccio). Phone: 215.898.0198; E-mail: lazar@mail.med. upenn.edu (M. Lazar).

ERC's present address is: Case Western School of Medicine, Cleveland, Ohio, USA.

JRD's present address is: Harvard Medical School, Boston, Massachusetts, USA.

SEM's present address is: Janssen Research and Development, Spring House, Pennsylvania, USA.

SRR's present address is: GlaxoSmithKline, Collegeville, Pennsylvania, USA.
1. Hardy OT, Czech MP, Corvera S. What causes the insulin resistance underlying obesity? Curr Opin Endocrinol Diabetes Obes. 2012;19(2):81-87.

2. Iozzo P. Viewpoints on the Way to the Consensus Session: where does insulin resistance start? The adipose tissue. Diabetes Care. 2009; 32(suppl 2):S168-S173.

3. Montague CT, O'Rahilly S. The perils of portliness: causes and consequences of visceral adiposity. Diabetes. 2000;49(6):883-888.

4. Ravussin E, Tataranni PA. Dietary fat and human obesity. JAm Diet Assoc. 1997;97(7 suppl):S42-S46.

5. Hariri N, Thibault L. High-fat diet-induced obesity in animal models. Nutr Res Rev. 2010;23(2):270-299.

6. Almind K, Kahn CR. Genetic determinants of energy expenditure and insulin resistance in diet-induced obesity in mice. Diabetes. 2004;53(12):3274-3285.

7. Gallou-Kabani C, et al. C57BL/6J and A/J mice fed a high-fat diet delineate components of metabolic syndrome. Obesity (Silver Spring). 2007;15(8):1996-2005.

8. West DB, Waguespack J, McCollister S. Dietary obesity in the mouse: interaction of strain with diet composition. Am J Physiol. 1995; 268(3 pt 2):R658-R665.

9. Lin C, et al. QTL analysis of dietary obesity in C57BL/6byj X 129P3/J F2 mice: diet- and sexdependent effects. PLoS One. 2013;8(7):e68776.

10. Su Z, Korstanje R, Tsaih SW, Paigen B. Candidate genes for obesity revealed from a C57BL/6J x 129S1/SvImJ intercross. Int J Obes (Lond). 2008;32(7):1180-1189.

11. Parks BW, et al. Genetic control of obesity and gut microbiota composition in response to high-fat, high-sucrose diet in mice. Cell Metab. 2013;17(1):141-152.

12. DeClercq VC, Goldsby JS, McMurray DN, Chapkin RS. Distinct adipose depots from mice differentially respond to a high-fat, high-salt diet.
J Nutr. 2016;146(6):1189-1196.

13. Weisberg SP, McCann D, Desai M, Rosenbaum M, Leibel RL, Ferrante AW. Obesity is associated with macrophage accumulation in adipose tissue. J Clin Invest. 2003;112(12):1796-1808.

14. Choi M-S, et al. High-fat diet decreases energy expenditure and expression of genes controlling lipid metabolism, mitochondrial function and skeletal system development in the adipose tissue, along with increased expression of extracellular matrix remodelling- and inflammationrelated genes. Br J Nutr. 2015;113(6):867-877.

15. Nadler ST, Stoehr JP, Schueler KL, Tanimoto G, Yandell BS, Attie AD. The expression of adipogenic genes is decreased in obesity and diabetes mellitus. Proc Natl Acad Sci U S A. 2000;97(21):11371-11376.

16. Voigt A, Agnew K, van Schothorst EM, Keijer J, Klaus S. Short-term, high fat feeding-induced changes in white adipose tissue gene expression are highly predictive for long-term changes. $\mathrm{Mol}$ Nutr Food Res. 2013;57(8):1423-1434.

17. Lehrke M, Lazar MA. The many faces of PPAR $\gamma$. Cell. 2005;123(6):993-999.

18. Soccio RE, Chen ER, Lazar MA. Thiazolidinediones and the promise of insulin sensitization in type 2 diabetes. Cell Metab. 2014;20(4):573-591.

19. Tomas J, et al. High-fat diet modifies the PPAR- $\gamma$ pathway leading to disruption of microbial and physiological ecosystem in murine small intestine. Proc Natl Acad Sci U S A. 2016;113(40):E5934-E5943.

20. Stienstra R, Duval C, Keshtkar S, van der Laak J, Kersten S, Müller M. Peroxisome proliferator-activated receptor gamma activation promotes infiltration of alternatively activated macrophages into adipose tissue. J Biol Chem. 2008;283(33):22620-22627.

21. Harms M, Seale P. Brown and beige fat: development, function and therapeutic potential. Nat Med. 2013;19(10):1252-1263.
22. Meierhofer D, Weidner C, Sauer S. Integrative analysis of transcriptomics, proteomics, and metabolomics data of white adipose and liver tissue of high-fat diet and rosiglitazone-treated insulin-resistant mice identified pathway alterations and molecular hubs. J Proteome Res. 2014;13(12):5592-5602.

23. Rong JX, et al. Adipose mitochondrial biogenesis is suppressed in $\mathrm{db} / \mathrm{db}$ and high-fat diet-fed mice and improved by rosiglitazone. Diabetes. 2007;56(7):1751-1760.

24. Callinan PA, Feinberg AP. The emerging science of epigenomics. Hum Mol Genet. 2006; 15(suppl 1):R95-R101.

25. Leung A, et al. Open chromatin profiling in mice livers reveals unique chromatin variations induced by high fat diet. J Biol Chem. 2014;289(34):23557-23567.

26. Kang $S$, et al. Identification of nuclear hormone receptor pathways causing insulin resistance by transcriptional and epigenomic analysis. Nat Cell Biol. 2015;17(1):44-56.

27. Heinz S, et al. Simple combinations of lineagedetermining transcription factors prime cis-regulatory elements required for macrophage and $\mathrm{B}$ cell identities. Mol Cell. 2010;38(4):576-589.

28. Pedersen DJ, et al. A major role of insulin in promoting obesity-associated adipose tissue inflammation. Mol Metab. 2015;4(7):507-518.

29. Lumeng CN, Deyoung SM, Bodzin JL, Saltiel AR. Increased inflammatory properties of adipose tissue macrophages recruited during dietinduced obesity. Diabetes. 2007;56(1):16-23.

30. Lefterova MI, et al. Cell-specific determinants of peroxisome proliferator-activated receptor gamma function in adipocytes and macrophages. Mol Cell Biol. 2010;30(9):2078-2089.

31. $\mathrm{Xu} \mathrm{H}$, et al. Chronic inflammation in fat plays a crucial role in the development of obesity-related insulin resistance. J Clin Invest. 2003;112(12):1821-1830. 
32. Deng T, et al. Class II major histocompatibility complex plays an essential role in obesityinduced adipose inflammation. Cell Metab. 2013;17(3):411-422.

33. Straus DS, Glass CK. Anti-inflammatory actions of PPAR ligands: new insights on cellular and molecular mechanisms. Trends Immunol. 2007;28(12):551-558.

34. Wilson-Fritch L, et al. Mitochondrial remodeling in adipose tissue associated with obesity and treatment with rosiglitazone. J Clin Invest. 2004;114(9):1281-1289.

35 . Wu J, et al. Beige adipocytes are a distinct type of thermogenic fat cell in mouse and human. Cell. 2012;150(2):366-376.

36. Vitali A, Murano I, Zingaretti MC, Frontini A, Ricquier D, Cinti S. The adipose organ of obesity-prone C57BL/6J mice is composed of mixed white and brown adipocytes. JLipid Res. 2012;53(4):619-629.

37. Kozak LP, Anunciado-Koza R. UCP1: its involvement and utility in obesity. Int JObes (Lond). 2008;32(suppl 7):S32-S38.

38. Norton N, et al. Universal, robust, highly quantitative SNP allele frequency measurement in DNA pools. Hum Genet. 2002;110(5):471-478.

39. Soccio RE, et al. Genetic variation determines PPAR $\gamma$ function and anti-diabetic drug response in vivo. Cell. 2015;162(1):33-44.

40. Haakonsson AK, Stahl Madsen M, Nielsen R, Sandelin A, Mandrup S. Acute genome-wide effects of rosiglitazone on PPAR $\gamma$ transcriptional networks in adipocytes. Mol Endocrinol. 2013;27(9):1536-1549.

41. Step SE, et al. Anti-diabetic rosiglitazone remodels the adipocyte transcriptome by redistributing transcription to PPAR $\gamma$-driven enhancers. Genes Dev. 2014;28(9):1018-1028.

42. Kozak LP. The genetics of brown adipocyte induction in white fat depots. Front Endocrinol (Lausanne). 2011;2:64.

43. Di Mascolo D, et al. Rosiglitazone-loaded nanospheres for modulating macrophage-specific inflammation in obesity. J Control Release. 2013;170(3):460-468.

44. Jilkova ZM, et al. Adipose tissue-related proteins locally associated with resolution of inflammation in obese mice. Int JObes (Lond). 2014;38(2):216-223.

45. Nguyen MT, et al. Regulation of chemokine and chemokine receptor expression by PPAR $\gamma$ in adipocytes and macrophages. PLoS One. 2012;7(4):e34976.

46. Kraakman MJ, et al. Blocking IL-6 trans-signaling prevents high-fat diet-induced adipose tissue macrophage recruitment but does not improve insulin resistance. Cell Metab. 2015;21(3):403-416.

47. Olefsky JM, Glass CK. Macrophages, inflammation, and insulin resistance. Annu Rev Physiol. 2010;72:219-246.

48. Marathe $\mathrm{C}$ et al. Preserved glucose tolerance in high-fat-fed C57BL/6 mice transplanted with PPAR $\gamma^{-/-}$, PPAR $\Delta^{-/-}$, PPAR $\gamma \Delta^{-/,}$, or LXR $\alpha \beta^{-/-}$bone marrow. J Lipid Res. 2009;50(2):214-224.

49. Blüher M. Adipose tissue inflammation: a cause or consequence of obesity-related insulin resistance? Clin Sci Lond Engl. 2016;130(18):1603-1614.

50. Zhang B, et al. Negative regulation of peroxisome proliferator-activated receptor- $\gamma$ gene expression contributes to the antiadipogenic effects of tumor necrosis factor- $\alpha$. Mol Endocrinol. 1996;10(11):1457-1466.

51. Jang MK, Jung MH. ATF3 represses PPAR $\gamma$ expression and inhibits adipocyte differentiation. Biochem Biophys Res Commun . 2014;454(1):58-64.

52. Ohno H, Shinoda K, Spiegelman BM, Kajimura S. PPAR $\gamma$ agonists induce a white-to-brown fat conversion through stabilization of PRDM16 protein. Cell Metab. 2012;15(3):395-404.

53. Walker GE, et al. Subcutaneous abdominal adipose tissue subcompartments: potential role in rosiglitazone effects. Obesity (Silver Spring). 2008;16(9):1983-1991.

54. Wang W, Seale P. Control of brown and beige fat development. Nat Rev Mol Cell Biol. 2016;17(11):691-702.

55. Guerra C, Koza RA, Yamashita H, Walsh K, Kozak LP. Emergence of brown adipocytes in white fat in mice is under genetic control. Effects on body weight and adiposity. JClin Invest. 1998;102(2):412-420.

56. Shabalina IG, Petrovic N, de Jong JM, Kalinovich AV, Cannon B, Nedergaard J. UCP1 in brite/beige adipose tissue mitochondria is functionally thermogenic. Cell Rep. 2013;5(5):1196-1203.

57. Xue B, Rim JS, Hogan JC, Coulter AA, Koza RA, Kozak LP. Genetic variability affects the development of brown adipocytes in white fat but not in interscapular brown fat. J Lipid Res. 2007;48(1):41-51

58. Coulter AA, Bearden CM, Liu X, Koza RA, Kozak LP. Dietary fat interacts with QTLs controlling induction of Pgc-1 $\alpha$ and Ucp1 during conversion of white to brown fat. Physiol Genomics. 2003;14(2):139-147.

59. Seale P, et al. PRDM16 controls a brown fat/skeletal muscle switch. Nature. 2008;454(7207):961-967.

60. Koza RA, Hohmann SM, Guerra C, Rossmeisl M, Kozak LP. Synergistic gene interactions control the induction of the mitochondrial uncoupling protein (Ucp1) gene in white fat tissue. J Biol Chem. 2000;275(44):34486-34492.

61. Li Y, Bolze F, Fromme T, Klingenspor M. Intrinsic differences in BRITE adipogenesis of primary adipocytes from two different mouse strains. Biochim Biophys Acta . 2014;1841(9):1345-1352.

62. Esterbauer H, et al. Uncoupling protein-1 mRNA expression in obese human subjects: the role of sequence variations at the uncoupling protein-1 gene locus. J Lipid Res. 1998;39(4):834-844.

63. Fumeron F, et al. Polymorphisms of uncoupling protein (UCP) and beta 3 adrenoreceptor genes in obese people submitted to a low calorie diet. Int J Obes Relat Metab Disord. 1996;20(12):1051-1054.

64. Heilbronn LK, Kind KL, Pancewicz E, Morris AM, Noakes M, Clifton PM. Association of -3826 G variant in uncoupling protein-1 with increased $\mathrm{BMI}$ in overweight Australian women. Diabetologia. 2000;43(2):242-244.

65. Kim KS, et al. The finding of new genetic polymorphism of UCP-1 A-1766G and its effects on body fat accumulation. Biochim Biophys Acta. 2005;1741(1-2):149-155.

66. Nicoletti CF, et al. UCP1 -3826 A>G polymorphism affects weight, fat mass, and risk of type 2 diabetes mellitus in grade III obese patients.
Nutrition. 2016;32(1):83-87.

67. Nagai N, Sakane N, Tsuzaki K, Moritani T. UCP1 genetic polymorphism $(-3826 \mathrm{~A} / \mathrm{G})$ diminishes resting energy expenditure and thermoregulatory sympathetic nervous system activity in young females. Int J Obes (Lond). 2011;35(8):1050-1055.

68. Yoneshiro T, et al. Impact of UCP1 and $\beta 3$ AR gene polymorphisms on age-related changes in brown adipose tissue and adiposity in humans. Int JObes (Lond). 2013;37(7):993-998.

69. Rose G, Crocco P, D’Aquila P, Montesanto A, Bellizzi D, Passarino G. Two variants located in the upstream enhancer region of human UCP1 gene affect gene expression and are correlated with human longevity. Exp Gerontol. 2011;46(11):897-904.

70. Brondani LA, Assmann TS, de Souza BM, Bouças AP, Canani LH, Crispim D. Meta-analysis reveals the association of common variants in the uncoupling protein (UCP) 1-3 genes with body mass index variability. PLoS One. 2014;9(5):e96411.

71. Fall T, Ingelsson E. Genome-wide association studies of obesity and metabolic syndrome. $\mathrm{Mol}$ Cell Endocrinol. 2014;382(1):740-757.

72. Deplancke B, Alpern D, Gardeux V. The genetics of transcription factor DNA binding variation. Cell. 2016;166(3):538-554.

73. Langmead B, Trapnell C, Pop M, Salzberg SL. Ultrafast and memory-efficient alignment of short DNA sequences to the human genome. Genome Biol. 2009;10(3):R25.

74. Saldanha AJ. Java Treeview - extensible visualization of microarray data. Bioinforma OxfEngl. 2004;20(17):3246-3248.

75. McLean CY, et al. GREAT improves functional interpretation of cis-regulatory regions. Nat Biotechnol. 2010;28(5):495-501.

76. Bailey TL, et al. MEME SUITE: tools for motif discovery and searching. Nucleic Acids Res. 2009;37(Web Server issue):W202-W208.

77. Trapnell C, et al. Differential gene and transcript expression analysis of RNA-seq experiments with TopHat and Cufflinks. Nat Protoc. 2012;7(3):562-578.

78. Anders S, Pyl PT, Huber W. HTSeq - a Python framework to work with high-throughput sequencing data. Bioinforma OxfEngl. 2015;31(2):166-169.

79. Love MI, Huber W, Anders S. Moderated estimation of fold change and dispersion for RNA-seq data with DESeq2. Genome Biol. 2014;15(12):550.

80. Huang da W, Sherman BT, Lempicki RA. Systematic and integrative analysis of large gene lists using DAVID bioinformatics resources. Nat Protoc. 2009;4(1):44-57.

81. Keane TM, et al. Mouse genomic variation and its effect on phenotypes and gene regulation. Nature. 2011;477(7364):289-294.

82. Li H, et al. The Sequence Alignment/ Map format and SAMtools. Bioinformatics. 2009;25(16):2078-2079.

83. Thorvaldsdóttir H, Robinson JT, Mesirov JP. Integrative Genomics Viewer (IGV): high-performance genomics data visualization and exploration. Brief Bioinformatics. 2013;14(2):178-192.

84. Gerhart-Hines Z, et al. The nuclear receptor Rev-erb $\alpha$ controls circadian thermogenic plasticity. Nature. 2013;503(7476):410-413. 